\title{
Les nouvelles technologies au service de la toxicologie clinique
}

\section{New technologies in clinical toxicology}

Laurence LABAT ${ }^{(1)}$, Michel LHERMITTE*(2)

(1) Laboratoire de Chimie Analytique, UFR des Sciences Pharmaceutiques, 3, place de la Victoire 33076 BORDEAUX Cedex

(2) Laboratoire de Biochimie et de Biologie Moléculaire, CHRU de Lille, Hôpital Calmette, Boulevard du Prof. Jules Leclercq - 59037 LILLE Cedex

*Auteur à qui adresser la correspondance : Pr. Michel LHERMITTE, Laboratoire de Biochimie et de Biologie Moléculaire, CHRU de Lille, Hôpital Calmette, Boulevard du Prof. Jules Leclercq - 59037 LILLE Cedex Tél : 0320444963 - Fax : 0320444729 - e-mail : mlhermitte@chru-lille.fr

(Reçu le 3 octobre 2000 ; accepté le 25 octobre 2000)

\section{RÉSUMÉ}

Depuis une dizaine d'années, l'Electrophorèse Capillaire (EC) et la spectroscopie de Résonance Magnétique Nucléaire $(R M N)$ sont entrées dans le domaine de la biologie clinique. Ces techniques peuvent être utilisées pour l'analyse de xénobiotiques dans les prélèvements biologiques. Cet article dresse le bilan actuel de l'apport de ces deux technologies pour la toxicologie clinique. Leurs intérêts respectifs dans le domaine de la toxicologie analytique, les progrès récents et des applications sont présentés. Afin de pouvoir identifier dans des conditions d'urgence les médicaments utilisés à des fins d'intoxications ou la prise de stupéfiants, l'EC et la RMN pourraient être des compléments intéressants aux méthodes chromatographiques largement utilisées.

\section{MOTS-CLÉS}

Electrophorèse capillaire, Résonance Magnétique Nucléaire, Toxicologie clinique.

\section{SUMMARY}

Last ten years, Capillary Electrophoresis $(C E)$ and Nuclear Magnetic Resonance (NMR) were introduced and used in clinical laboratory. These technics can be used to detect xenobiotics in biological fluids. This paper is a review of these two technics in analytical toxicology. Applications are described. CE and NMR could be complementary methods to conventional chromatography in a toxicological laboratory.

\section{KEY-WORDS}

Capillary electrophoresis, Nuclear Magnetic Resonance, Clinical Toxicology. 


\section{Introduction}

Depuis quelques années, la recherche et l'identification des xénobiotiques en toxicologie clinique prend une place de plus en plus grande. Les méthodes colorimètriques ont été supplantées par des méthodes immunoenzymatiques de recherche de médicaments. Si celles-ci sont rapides, elles ne permettent de mettre en évidence que certains médicaments ou certaines classes de médicaments (barbituriques, benzodiazépines, antidépresseurs tricycliques) ou de stupéfiants (dérivés opiacés, amphétamines). De plus, dans une même classe médicamenteuse, certaines molécules ne sont pas ou sont peu détectées (1). Des méthodes chromatographiques de recherche peuvent également, après extraction plus ou moins spécifique des molécules, mettre en évidence celles qui sont responsables d'intoxications aiguës. Ainsi la chromatographie en phase gazeuse (CPG) couplée avec différentes détections (capture d'électrons, SM...) (2) et la chromatographie liquide haute performance (CLHP) couplée à différentes détections (DBD, fluorimétrie, SM...) (3), sont de plus en plus utilisées.

A côté de ces différentes méthodes, sont apparues depuis une dizaine d'année, l'électrophorèse capillaire (EC) et la spectroscopie de résonance magnétique nucléaire (RMN). Ces dernières sont encore au stade du développement et pourraient trouver leur place dans le domaine de la toxicologie clinique en tant que méthodes complémentaires.

\section{Utilisation de} l'Electrophorèse Capillaire en toxicologie clinique

L'EC est devenue commercialement accessible et a permis le développement de nombreuses méthodes pour l'analyse d'un nombre croissant de xénobiotiques dans les milieux biologiques (4). Son pouvoir séparatif très important, sa sensibilité suffisante font de cette technologie un outil très prometteur.

Nous décrirons dans cette première partie des applications des différentes technologies en EC adaptées au dosage de médicaments et de stupéfiants dans les milieux biologiques (sang, urine). Malheureusement, ces applications n'ont pas toujours été optimisées dans le but d'une utilisation dans un laboratoire d'urgence. Nous les avons classé en fonction de l'intérêt principal que présente l'EC par rapport à l'analyse classique en toxicologie.

\section{Préparation minimale de l'échantillon et détection optique}

En EC, les milieux biologiques peuvent être analysés par injection directe sans prétraitement ou après une préparation minimale : simple dilution, centrifugation, filtration, ultrafiltration ou précipitation protéique (5). En EC en mode micellaire (CECM), l'effet dénaturant du dodécyl sulfate de sodium sur les protéines du sérum permet l'injection directe. Ce mode d'injection reste cependant peu utilisé en raison de la faible concentration des molécules recherchées dans les échantillons. Il a permis l'analyse de sérum ou d'urine de patients dans des cas de suspicions de surdosages à des antalgiques (paracétamol, acide acétylsalicylique, ibuprofêne, naproxène, kétoprofêne) (6) ou à des anticonvulsivants (carbamazépine, phénobarbital) (7). Dans ces deux études, les auteurs ne précisent pas les limites de détection. Wu (8) réalise la détection de la morphine et de la morphine 3- $\beta$-D-glucuronide en EC de zone (ECZ) après injection directe d'urines acidifiées à $\mathrm{pH}$ 2-3. Malheureusement, les limites de détection obtenues sont très élevées (voir tableau I) et dans ces conditions l'EC 'est une alternative peu intéressante à un screening pouvant être réalisé en immunoanalyse (8).

Les modes de détection les plus simples et les plus couramment utilisés en EC sont les détections optiques : ultraviolet (UV) ou fluorescence induite par laser (FIL) (9). En raison d'un trajet optique très court (la détection étant directement réalisée en ligne sur une fenêtre du capillaire), les concentrations minimales détectables en UV sans préconcentration de l'échantillon sont de l'ordre du $\mu \mathrm{g} / \mathrm{mL}$. Comparée à la détection UV, la détection par FIL permet une nette augmentation de la sensibilité (100 fois). Des concentrations urinaires thérapeutiques de zopiclone, zolpidem et de leurs métabolites principaux ont été déterminées en EC-FIL (sans extraction des échantillons) $(9,10)$. Leur séparation en 10 minutes a permis d'obtenir des limites de détection de l'ordre de $2 \mathrm{ng} / \mathrm{mL}$. Cependant, la détection par FIL bien que plus sensible que le mode UV nécessite un appareillage plus complexe et les longueurs d'ondes d'excitation sont limitées à $325 \mathrm{~nm}$ pour le laser $\mathrm{He}-\mathrm{Cd}$ et $488 \mathrm{~nm}$ pour le laser Ar. Elle ne peut donc pas encore être considérée comme une détection utilisée en routine pour la détermination des médicaments dans les milieux biologiques (9).

\section{Concentration par une étape d'extrac- tion ou un effet de stacking}

Dans de nombreux cas, l'analyse des xénobiotiques dans les milieux biologiques par injection directe ou 
après traitement simplifié de l'échantillon est impossible. Des étapes d'extraction, voire de concentration s'avèrent indispensables (4). En parallèle, on réalise en EC une préconcentration de l'échantillon en ligne en utilisant le mode d'injection électrocinétique et les procédures d'amplification de champ électrique nommées stacking effects (5). Les exemples sont nombreux dans la littérature (voir tableau I). On citera l'analyse après extraction des dérivés opioides dans le plasma ou les urines (11), des antidépresseurs tricycliques dans le plasma par CECM (12), des benzodiazépines dans les urines par CECM (13), des barbituriques, du paracétamol et des salicylés dans le sérum et les urines par ECZ (14).

La sensibilité de l'EC reste un facteur limitant pour que cette technique puisse être comparée aux techniques chromatographiques. Plusieurs stratégies classiquement utilisées en EC peuvent cependant contribuer à son amélioration : utilisation du mode d'injection électrocinétique, allongement du temps d'injection à l'origine d'un effet d'amplification du champ électrique (large volume sample stacking, LVSS), concentration de l'échantillon lors de l'injection par diminution de la concentration en sels dans l'échantillon ou pré-injection d'un bouchon d'eau ou de solvant $(5,9)$. Taylor $(15)$ a démontré que l'on pouvait abaisser la limite de détection des opiacés dans des extraits urinaires en privilégiant le mode d'injection électrocinétique par rapport au mode hydrodynamique. Wey (11) décrit une méthode d'amplification de champ électrique en tête de colonne avec une pré-injection qui permet de détecter les dérivés opiacés. A partir d'extraits de faibles volumes de prélèvements de plasma, sang ou urine (20 à $100 \mu \mathrm{L}$ ), les molécules sont détectables jusqu'à des concentrations de l'ordre du $\mathrm{ng} / \mathrm{mL}$ en détection UV. Le choix des faibles volumes permet de réduire à son minimum la concentration en sels dans l'échantillon injecté améliorant ainsi les limites de détection par stacking effect.

Il est donc important de réaliser que les limites de la sensibilité de l'EC ne sont pas seulement dues au type de détecteur utilisé, mais dépendent également du mode d'injection et de la matrice de l'échantillon injecté.

\section{Développement de screening en électro- phorèse capillaire}

Quelques études en EC présentent des méthodes spécifiques pour le screening de plusieurs classes de molécules après extraction dans les milieux biologiques (4, 16-19). D'autres méthodes ont été développées, permettant la séparation de différentes molécules d'une même classe médicamenteuse contenues dans des mélanges étalons. Pucci (20) sépare ainsi 11 antidépresseurs tricycliques et phénothiazines et Boone (21) évalue différentes techniques en EC pour optimiser la séparation de 25 dérivés barbituriques (voir tableau I). Dans le cadre d'un screening médicamenteux appliqué en toxicologie d'urgence sur des prélèvements urinaires provenant de patients d'unités de soins intensifs, Thormann (19) a développé puis appliqué en routine des techniques électrophorétiques en mode ECZ ou CECM. Une extraction en phase solide des urines est réalisée en deux étapes, permettant la séparation de molécules acide, neutre ou basique. La spectrométrie par barrette de diodes permet la détection de barbituriques, méthadone, dérivés du cannabis, benzodiazépines, opiacés, cocaïne, et amphétamines $(18,19)$. Les limites de sensibilité obtenues après extraction de $5 \mathrm{~mL}$ d'urine sont de l'ordre de $50 \mathrm{ng} / \mathrm{mL}$. Ainsi, le flunitrazépam non détecté en immunoanalyse, peut être identifié en CECM. Après ingestion de $1 \mathrm{mg}$ de flunitrazépam et recueil des urines pendant 8 heures, l'analyse du prélèvement après hydrolyse ( $\beta$-glucuronidase/arylsulphatase) et extraction en phase solide met en évidence une concentration de $300 \mathrm{ng} / \mathrm{mL}$ pour le métabolite urinaire principal, le 7-aminoflunitrazépam (19).

L'EC reste cependant peu utilisée en routine pour des screening en toxicologie (17). En 1998, Hudson a développé une méthode générale de screening dans le sang, de molécules d'intérêt médico-légal (22). Cette banque de données permet l'identification de 650 composés.

Afin de pouvoir standardiser et transposer les méthodologies en EC d'un laboratoire à l'autre, il est essenciiel de définir des paramètres d'identification précis pour chaque molécule. Malheureusement, l'instrumentation n'est pas encore standardisée (modes d'injections différents, différences de position de la cellule de détection sur le capillaire). Ceci est responsable du manque de reproductibilité des paramètres de migration (23). De plus, les tensions non constantes appliquées pendant l'analyse, certaines interactions entre les électrolytes et la paroi du capillaire et les variations du flux électroosmotique (FEO) s'ajoutent aux variations dues à l'instrumentation. En analyse quantitative, l'ajout d'un étalon interne corrige les fluctuations du volume d'injection, mais pour l'analyse qualitative, il est important de pouvoir choisir un paramètre d'identification fiable. Le manque de reproductibilité du temps de migration étant connu, on lui préfère la mobilité électrophorétique efficace. Ce paramètre est indépendant du flux électroosmotique et beaucoup plus reproductible que le temps de migration $(21,23)$

Boone utilise la mobilité électrophorétique efficace comme paramètre d'identification de 25 barbituriques 
différents. La répétabilité des mobilités pour cette étude est ainsi tout à fait satisfaisante, avec des valeurs inférieures à $0,6 \%$ (21). En utilisant ce nouveau paramètre comme critère d'identification, la reproductibilité de la méthodologie est nettement améliorée. La standardisation des appareils et le contrôle des mobilités une fois maîtrisées, on peut concevoir une adaptation correcte de ce type de screening en analyse de routine. Boone décrit également l'absence de corrélation entre le screening réalisé en EC et celui réalisé par une technique chromatographique et conclut sur l'intérêt pour les laboratoires de toxicologie de posséder une combinaison de ces technologies.

\section{Analyse des ions en électrophorèse capil- laire}

De très nombreux anions peuvent être responsables d'intoxications. Le choix des méthodes de dosage reste cependant très limité (24). Les techniques colorimétriques, la chromatographie d'échanges d'ions, le dosage par des électrodes spécifiques ou la CPG-SM sont les techniques les plus couramment utilisées (24). Le dosage des ions en EC permet des séparations simples et rapides. Les ions migrent sous forme de bandes étroites par effet de stacking. On peut observer des pics très fins et d'une très grande efficacité (parfois près de 1 million de plateaux théoriques) (25). Fritz (26) décrit des séparations de mélanges standards de 8 ou 10 anions en 6 minutes.

La grande majorité des analyses d'ions décrites en EC sont réalisées en mode de détection UV direct ou indirect sur des échantillons d'eau ou des matrices très propres (27). Les ions bromures, sulfates, nitrites, nitrates, fluorures et phosphates sont détectés en UV indirect dans de l'eau ou des solutions aqueuses. Le mode d'injection électrocinétique, très sélectif pour les 'ions, a été choisi pour améliorer la sensibilité. Il permet la détection de concentrations très faibles (limites de détection de 0,2 à $1 \mathrm{ng} / \mathrm{mL}$ ), nécessaire dans ce type d'application (27). Lorsque l'analyse doit être réalisée dans des prélèvements biologiques, le choix de ce mode d'injection pose un problème. En raison de l'effet de la matrice sur l'injection électrocinétique, on lui préfère l'injection hydrodynamique (27).

Peu d'études décrivent le dosage des ions en EC dans les milieux biologiques. A l'heure actuelle, les anions les plus étudiés sont les nitrites et les nitrates, marqueurs dans le plasma de la production de NO $(25,28,29)$. Bories (29) sépare dans le sérum les deux anions en moins de 4 minutes, avec une limite de détection de 10 $\mu \mathrm{mol} / \mathrm{L}$ en UV $(214 \mathrm{~nm})$. La technique est beaucoup plus rapide que pour les techniques chromatographiques
( 15 min.) ou enzymatiques ( 45 min.). La sensibilité est satisfaisante, du même ordre de grandeur que celles décrites en chromatographie échangeuses d'ions (24). Dans cette étude, les sérums sont injectés directement après dilution dans de l'eau. Les protéines du sérum interfèrent avec les ions et les parois du capillaire mais permettent cependant la réalisation très rapide de dosages reproductibles (29). Dans d'autres études, on déprotéinise l'échantillon par ajout d'acétonitrile (25). En 1999, Bories critique cette étape préparative en démontrant qu'elle n'est pas indispensable et qu'elle est source de contaminations pour l'échantillon (29).

Très récemment, une méthode originale a été décrite pour l'analyse des ions. Elle couple le principe de la chromatographie échangeuse d'ions et de l'EC dans une seule technique (26). Les ions sont séparés en fonction de leur mobilité électrophorétique et de leur pouvoir d'échange avec un polymère dissout dans le tampon de migration. Fritz décrit ainsi la séparation de 17 anions organiques et inorganiques en moins de 6 minutes (26). Macka (30) décrit dans une revue de la littérature toutes les applications de l'EC pour la détermination des ions métaux. Quelques unes des études ont permis l'analyse des ions $\mathrm{Pb}, \mathrm{Cd}, \mathrm{Hg}, \mathrm{Ni}, \mathrm{Li}, \mathrm{Sr}$, et $\mathrm{Al}$ dans le sérum. Une détection directe en UV permet l'analyse après la formation de complexes avec les différents ions. Malheureusement, les limites de détection ne sont pas précisées.

L'EC a un pouvoir de séparation très important pour les mélanges complexes de cations et d'anions. Les séparations sont rapides et nécessitent peu de prélèvements. Une détection en mode UV indirect est utilisé assez couramment pour la détection d'une majorité d'ions. Cependant, si l'EC semble tout à fait adaptée à l'analyse des ions, peu de méthodes ont été développées dans les milieux biologiques. Les avantages de cette technique pour le dosage des ions susciteront certainement des développements dans ces matrices plus complexes.

\section{Screening des substances illicites et ana- lyse chirale}

Le potentiel de l'EC pour la séparation chirale est important : par simple addition d'un séparateur chiral comme les cyclodextrines dans le tampon de migration, l'EC représente une méthode simple, peu coûteuse et efficace pour la séparation des énantiomères (31). Aujourd'hui, il n'existe pas d'application de séparations chirales étudiées en toxicologie clinique. Cependant, plusieurs études montrent l'intérêt pour le diagnostic toxicologique de la séparation et du dosage de certaines formes énantiomèriques, comme par exemple dans le cas de la méthadone (32). 
On citera également les études réalisées dans le cadre de la lutte contre le dopage pour des groupes de substances interdites. Les deux classes de médicaments ayant fait l'objet d'études dans ce domaine sont les diurétiques $(17,33)$ et les $\beta$ bloquants (34) dans le sang et les urines. Dans le contexte du dopage, une séparation des formes énantiomèriques de certaines molécules s'est déjà révélée intéressante lorsqu'une seule des deux formes est une substance interdite alors que l'autre est une substance autorisée dotée de propriétés pharmacologiques. Un des exemples décrits est la séparation de la forme $(+)$ du propoxyphène, substance narcotique d'utilisation contrôlée et du (-) propoxyphène, antitussif autorisé (31).

\section{Combinaison de l'électrophorèse capil- laire et de l'immunoanalyse}

Très récemment, la combinaison de la technique électrophorétique et de l'immunoanalyse a suscité une attention particulière. Le principal intérêt de ce nouveau concept est de pouvoir réaliser le dépistage ou le dosage de plusieurs molécules dans les urines sans passer par l'étape d'extraction. Il nécessite de très faibles volumes de prélèvement (20 à $50 \mu \mathrm{L})$, une incubation préalable de l'anticorps et du traceur et une séparation $\mathrm{du}$ traceur fluorescent libre et du complexe anticorps/traceur en ECZ ou en CECM avec une détection en mode FIL $(5,31)$. A l'heure actuelle, l'association de ces deux techniques est encore peu utilisée pour l'étude des médicaments et l'équipe de Thormann est une des seules à notre connaissance travaillant sur ce concept (19, 35-37). Des limites de détection de l'ordre de $30 \mathrm{ng} / \mathrm{mL}$ pour un dosage urinaire simultané de la méthadone, de dérivés des opiacés, de la benzoylecgonine et des amphétamines sont décrites (36). Les résultats obtenus sont tout à fait comparables à ceux des techniques EMIT ou FPIA classiquement utilisées dans les screenings en toxicologie. D'autres exemples peuvent être cités comme le dosage simultané de l'acide salicylique et du paracétamol ou celui du paracétamol, de la théophylline et de la quinidine dans le sérum (35).

Cette technique dans le cadre d'une utilisation en toxicologie d'urgence présente les avantages d'être simple, rapide et suffisamment sensible. C'est une approche originale pour rechercher plusieurs xénobiotiques simultanément dans un milieu biologique. Ce concept semble offrir de nombreuses possibilités d'application, il garde néanmoins les inconvénients spécifiques des techniques immunologiques.

\section{Confirmation par électrophorèse capil- laire couplée à la spectrométrie de masse}

Dans un effort d'analogie avec la CPG-SM ou la CLHP-SM, reconnues de façon universelle, le couplage de l'EC et de la spectromètrie de masse (EC-SM) apparaît comme une nouvelle technologie dans le domaine de la toxicologie. Quelques exemples ont été récemment publiés pour l'identification et le dosage de médicaments : paracétamol et ses principaux métabolites dans les urines et le sérum (38), anti-inflammatoires (acide acétylsalicylique, ibuprofène, naproxène, kétoprofène) dans l'urine (6). On citera également des études réalisées pour confirmer la présence d'amphétamines, de designer drugs (39) ou de morphine après hydrolyse acide et extraction en phase solide des prélèvements urinaires (40). Récemment, McClean (41, 42) étudie la séparation théorique d'un mélange de 15 benzodiazépines. Une optimisation du couplage EC-SM permet l'amélioration de la reproductibilité de la méthode et l'abaissement des limites de détection (voir tableau I).

Bien que la plupart des études en EC-SM aient été réalisées avec d'autres objectifs, on peut très bien concevoir l'utilisation de ce couplage EC-SM en tant que méthode d'analyse en toxicologie d'urgence au même titre que la CPG-SM ou la CLHP-SM. Cependant, le développement de nouvelles interfaces et le prix de l'appareillage sont autant de paramètres qui freinent l'utilisation de l'EC-SM dans les laboratoires de toxicologie. Les avantages de l'utilisation de ce couplage, voire même d'un couplage EC-SM-SM $(39,40,42)$ dans l'identification des substances et une recherche très active dans ce domaine permettront certainement l'amélioration des systèmes et de leur utilisation.

\section{Conclusion}

Le pouvoir de séparation remarquable, la rapidité d'analyse, la versatilité, l'automatisation, et le faible coût en consommables sont des paramètres permettant de présenter l'EC comme une bonne alternative à d'autres techniques séparatrices consommatrices de temps et chères. L'EC est une technique reproductible, efficace et suffisamment sensible pour qu'elle soit adaptée à la séparation et à la quantification des xénobiotiques dans les milieux biologiques.

Les problèmes de reproductibilité des paramètres d'identification dans le cadre de screening semble être bien résolus avec l'utilisation de la mobilité électrophorétique efficace (23). L'efficacité de l'EC dans la séparation chirale reste à exploiter dans le domaine toxicologique. Depuis peu de temps, les applications de l'EC 
Tableau I : Étude en EC des xénobiotiques.

\begin{tabular}{|c|c|c|c|c|c|}
\hline Xénobiotiques & Matrice & $\begin{array}{c}\text { Procédure } \\
\text { d'extraction }\end{array}$ & L.d.d. & $\begin{array}{c}\text { Temps } \\
\text { d'analyse } \\
\end{array}$ & Références \\
\hline Anti-inflammatoires & Urine & ID & n.d & $<10 \mathrm{~min}$ & (6) \\
\hline $\begin{array}{l}\text { Morphine et M3ßD } \\
\text { glucuronide }\end{array}$ & Urine & ID & $\begin{array}{c}200 \mathrm{ng} / \mathrm{mL}(\mathrm{M})(\mathrm{DBD}) \\
500 \mathrm{ng} / \mathrm{mL} \text { (M glucur) }\end{array}$ & $15 \mathrm{~min}$. & (8) \\
\hline Dérivés opioïdes & $\begin{array}{l}\text { Plasma } \\
\text { Urine }\end{array}$ & EPS & $1 \mathrm{ng} / \mathrm{mL}$ (UV) & $<8$ min. & (11) \\
\hline Anti-inflammatoires & $\begin{array}{l}\text { Solution } \\
\text { Étalon }\end{array}$ & - & n.d & $<8 \mathrm{~min}$ & (43) \\
\hline $\begin{array}{l}25 \text { barbituriques } \\
\text { (screening) }\end{array}$ & $\begin{array}{c}\text { Solution } \\
\text { Étalon }\end{array}$ & - & n.d & $<5 \mathrm{~min}$ & (21) \\
\hline $\begin{array}{l}\text { Morphine et } \\
\text { amphétamine }\end{array}$ & Urine & EPS & $\begin{array}{c}\text { (M) } 50 \mathrm{ng} / \mathrm{mL} \text { ou } \\
10 \mathrm{ng} / \mathrm{mL} \text { en LVSS } \\
\text { (A) } 30 \mathrm{ng} / \mathrm{mL}(\mathrm{SM}-\mathrm{SM})\end{array}$ & $10 \mathrm{~min}$. & (40) \\
\hline 11 antidépresseurs & $\begin{array}{l}\text { Solution } \\
\text { Étalon }\end{array}$ & - & n.d & $10 \mathrm{~min}$. & (20) \\
\hline 15 benzodiazépines & $\begin{array}{c}\text { Solution } \\
\text { Étalon }\end{array}$ & - & $\begin{array}{c}410^{-6} \text { à } 510^{-7} \mathrm{~mol} / \mathrm{L} \\
(\mathrm{SM}-\mathrm{SM})\end{array}$ & $<15 \mathrm{~min}$ & $(41,42)$ \\
\hline $\begin{array}{l}\text { méthadone, opiacés } \\
\text { benzoylecgonine, } \\
\text { amphétamines }\end{array}$ & Urine & $\begin{array}{l}\text { Immuno- } \\
\text { Analyse }\end{array}$ & $>30 \mathrm{ng} / \mathrm{mL}$ (FIL) & $11 \mathrm{~min}$. & (36) \\
\hline
\end{tabular}

Avec L.d.d. - limite de détection, ID = injection directe, n.d = non déterminé, EPS = extraction en phase solide, $\mathrm{DBD}=$ détection en barrette de diodes, $\mathrm{SM}=$ spectrométrie de masse, $\mathrm{FIL}=$ Fluorescence induite par laser, $\mathbf{M}=$ morphine, $\mathrm{A}=$ amphétamine et $L V S S=$ large volume sample stacking.

en milieu non aqueux se développent, permettant de séparer les molécules de structures très homogènes difficilement séparables en ECZ ou en CECM (43). D'autres avantages s'ajoutent au choix de cette nouvelle technique : un tampon tout à fait adapté à un couplage à la SM, une bonne combinaison avec une étape d'extraction utilisant des solvants organiques (21), la possibilité de travailler sur des capillaires très courts permettant ainsi de réduire les temps d'analyses (entre 1 et $3 \mathrm{~min}$ ). Cependant, il faut rester prudent quant à la reproductibilité d'une analyse quantitative dans ces conditions en raison des problèmes d'évaporation des solvants organiques utilisés. L'EC possède de nombreuses caractéristiques intéressantes pour qu'elle puisse être utilisée un jour dans les laboratoires de toxicologie clinique en urgence. Peu de travaux existent dans ce domaine. De nombreux développements et une amélioration de l'instrumentation sont encore nécessaires à son essor.

\section{Utilisation de la Résonance Magnétique Nucléaire en biologie et en toxicologie clinique}

En médecine, les techniques de Résonance Magnétique Nucléaire (RMN) sont de plus en plus utilisées dans le domaine de l'imagerie (IRM) mais bien qu'elles permettent la caractérisation et l'analyse de très nombreuses molécules, l'étude des liquides biologiques est restée longtemps en dehors du champ des applications médicales courantes.

La RMN, technique spectroscopique, n'a pas la prétention de remplacer les différentes techniques déjà mises en œuvre dans un laboratoire de biologie clinique (1-3), mais d'être une méthode complémentaire dans cette démarche toxicologique. Elle a l'avantage en effet de ne 
pas demander de préparation d'échantillons, elle peut être très rapide et permet de déterminer la structure précise et de quantifier les molécules responsables d'une intoxication aiguë.

Cette technique est basée sur la propriété des noyaux de certains atomes qui placés dans un champ magnétique intense absorbent une certaine quantité d'énergie.

Le noyau le plus étudié est le proton car il possède une bonne sensibilité en RMN et il est présent dans la plupart des molécules organiques. Chaque type de proton $\left(-\mathrm{CH}_{3},-\mathrm{CH}_{2}\right.$ - et aromatique) d'une molécule va interagir différemment avec le champ magnétique de l'aimant et avec les autres protons de la molécule donnant un déplacement chimique et des couplages différents pour chaque proton (réalisation d'un spectre $\mathrm{RMN}^{1} \mathrm{H}$ ). A partir de l'étude de ces spectres, il est possible de retrouver la structure de la molécule et de déterminer sa concentration (44).

Il faut aussi signaler que l'étude des autres noyaux comme ${ }^{2} \mathrm{H},{ }^{13} \mathrm{C},(45){ }^{15} \mathrm{~N}$ et ${ }^{19} \mathrm{~F}(46,47)$ peut-être très intéressante en particulier avec les molécules marquées.

Pour l'étude des liquides biologiques qui sont des mélanges complexes en solution dans l'eau, on utilise des séquences RMN spécifiques qui permettent d'une part de réduire le signal de l'eau présente en concentration très importante et d'autre part, d'obtenir des informations lorsque l'on a superposition de signaux.

La spectroscopie de RMN des liquides biologiques couvre des domaines de plus en plus divers allant de la recherche fondamentale à l'investigation clinique courante.

\section{Applications biologiques}

La RMN a d'abord été utilisée pour identifier et quantifier des métabolites endogènes de faible poids moléculaire dans l'urine chez l'homme (48-50) ou pour identifier des composés organiques qui s'accumulent dans les urines d'enfants atteints d'enzymopathies (acidémie isovalérique ou méthylmalonique) $(51,52)$.

De nombreux liquides biologiques ont fait l'objet d'investigation par spectroscopie RMN du proton $(53,56)$. L'urine et le plasma sanguin sont les échantillons biologiques les plus étudiés. Quelques études se rapportent au liquide céphalorachidien, au liquide séminal, au liquide amniotique, au liquide synovial, à la bile et aux sécrétions lacrymales.

D'autres applications médicales de la RMN concernent le suivi des cancers (57) et des greffes d'organes (58, 59).

\section{Applications pharmaco-toxicologiques}

La spectroscopie RMN comme outil d'étude en pharmacologie et en toxicologie fait l'objet d'un développement important au sein de l'équipe de Nicholson (48). Les études portent sur la recherche, l'identification et le métabolisme de divers xénobiotiques et de leurs catabolites présents dans l'urine et dans la bile $(60,61)$, ainsi que la détermination de marqueurs spécifiques en toxicologie (62).

La mise en évidence de xénobiotiques dans les liquides biologiques demande en premier lieu de bien connaître les constituants normaux "physiologiques" de ces liquides biologiques.

Les spectres 'H-RMN des urines sont maintenant bien connus et publiés $(48,50)$. En comparant les spectres des sujets intoxiqués avec ceux d'urines de sujets ne prenant pas de médicaments, avec des spectres de standards ou avec des spectres déjà publiés dans la littérature, il est possible de mettre en évidence des xénobiotiques $(48,50,63,64)$. En effet, les xénobiotiques et leurs métabolites présentent en RMN, plusieurs résonances caractéristiques (multiplets) qui sont bien distincts de ceux des métabolites endogènes (65). La plupart du temps, les spectres sont comparés avec ceux des composés standards connus, ou lorsque les standards ne sont pas disponibles, les spectres sont assez caractéristiques pour permettre leur identification (65). Dans certains cas, il suffit de réaliser des surcharges avec le composé standard et de ré-analyser l'urine par RMN pour confirmer la présence du composé. On peut aussi réaliser des spectres RMN en deux dimensions (66) ou utiliser des séquences particulières d'excitation sélective de type TOCSY (67) pour améliorer les attributions des protons.

\section{Mise en évidence de médicaments par RMN}

Le paracétamol constitue un très bon exemple d'application de la RMN du proton. Les différents catabolites de cette substance : dérivés glucuronoconjugués, sulfatés, $\mathrm{N}$-acétyl-cystéinyl et cystéinyl sont détectés dans l'urine de sujets intoxiqués et les proportions de ces divers catabolites ont pu être évalués (68). Une autre étude a montré l'existence d'un cycle futile de déacétylation du paracétamol en 4-aminophénol, intermédiaire néphrotoxique, avant son élimination urinaire (69).

L'aspirine et ses métabolites peuvent aussi être facilement détectés et quantifiés dans l'urine par spectroscopie 'H-RMN (70). Maschke et coll. ont même pu montrer dans le cas d'une intoxication aiguë, que la forme pharmaceutique à l'origine de l'intoxication était de l'acétylsalicylate de lysine (Aspegic ${ }^{\circledast}$ ) (66). 
D'autres anti-inflammatoires comme l'ibuprofène, le naproxéne et leurs métabolites sont aussi mis en évidence dans les urines $(64,65)$.

La chloroquine et son principal métabolite, la monodeséthylchloroquine, ont également été identifiés par RMN dans les urines d'un patient intoxiqué par cet antipaludéen $(67,71)$.

Des antiépileptiques ont été détectés dans l'urine de sujets victimes d'une intoxication aiguë. C'est le cas de la carbamazépine (65) et de l'acide valproique $(65,72$, 73), dont les métabolites sont excrétés en grande quantité dans l'urine. Pour l'acide valproique, la RMN permet l'identification d'un dérivé glucuronoconjugué (72).

Komoroski et coll. ont également mis en évidence de l'érythromycine dans des urines, un macrolide polaire qu'ils ne détectaient pas en CPG-SM dans leur échantillon (65). Il avait également été possible de mettre en évidence les métabolites urinaires de l'ampicilline chez le rat et de montrer la présence d'un nouveau métabolite par RMN (74).

De même la pseudoéphédrine a pu être mise en évidence par Komoroski et coll (65) dans les urines d'une jeune fille de 16 ans. D'autres substances comme la diphenhydramine, la chlorpromazine, la caféine ont été également détectées par RMN (65).

Par contre en ce qui concerne d'autres médicaments comme les antipsychotiques (chlorpromazine et halopéridol) ou les antidépresseurs tricycliques (imipramine et amitryptiline), leur identification urinaire n'a pas été possible alors que la CPG-SM les mettait facilement en évidence (65). Pour ces composés il était observé en RMN des signaux détectables dans la région 6-8ppm, où apparaissent également des signaux correspondant à des métabolites endogènes aromatiques comme l'acide hippurique (65). Les spectres sont alors compliqués puisque des signaux correspondant aux métabolites endogènes et à certains xénobiotiques se superposent. Certaines résonances de xénobiotiques peuvent être cachées sous les signaux de composés endogènes. Les spectres sont donc difficiles à interpréter à basse résolution et notamment à $300 \mathrm{MHz}$.

\section{Mise en évidence d'autres xénobiotiques}

La RMN est capable de mettre en évidence des substances de faibles masses moléculaires telles que le propylène glycol (65), l'éthylène glycol (75), les alcools : éthanol, méthanol $(75,76)$, isopropanol $(76)$, l'acétone (76) ou les solvants tels que le tétrahydrofuranne (77), ainsi que d'autres composés de faible masse moléculaire tels que les acides formique et glycollique (75).
La RMN a également permis de mettre en évidence dans des urines directement sans extraction deux herbicides : le paraquat et le glyphosate $(78,79)$.

\section{Conclusion}

La RMN trouve son utilité dans l'identification d'un xénobiotique et/ou de ses métabolites quand ils sont excrétés dans l'urine en grande quantité, comme c'est le cas dans les intoxications aiguës. Cette technique ne demande pas de préparation de l'échantillon (quelquefois, les échantillons sont lyophilisés), ni de séparation chromatographique. Komoroski et coll. ont comparé cette technique avec la CPG-SM (65) et ont montré la possibilité d'identifier des médicaments et leurs métabolites sans préparation de l'échantillon. Cependant, cette technique ne donne pas des résultats tout à fait comparables à la CPG-SM et il y a dans leur expérience, $56 \%$ de concordance entre les deux méthodologies. La RMN identifiait le paracétamol, l'ibuprofène, l'aspirine, l'acide valproique, la carbamazépine et la pseudoéphédrine, par mise en évidence de la molécule mère ou de ses métabolites. Pour un patient, les résultats de la CPG-SM étaient négatifs, alors que la RMN mettait en évidence de l'érythromycine. La RMN était plus performante pour identifier les médicaments analgésiques et antiépileptiques, par contre les médicaments antipsychotiques ou antidépressurs tricycliques n'étaient pas mis en évidence par RMN. Au contraire de la CPG$\mathrm{SM}$, la RMN identifiait le propyléne glycol, composé retrouvé dans des formulations médicamenteuses, aussi bien que dans des cosmétiques, des lotions ou des pommades. La mise en évidence de ce composé peut signaler une prise de médicament (80) ou une intoxication aiguë par le propyléne glycol (81).

La spectroscopie RMN est une technique qui n'est pas encore facilement accessible au laboratoire de toxicologie. Elle est utile dans la mise en évidence des métabolites d'un xénobiotique, comme le métabolite hépatotoxique du paracétamol. Globalement, la CPG-SM s'avère aujourd'hui plus utile que la RMN pour identifier le produit responsable d'une intoxication aiguë. Cependant avec cette technique l'échantillon est détruit alors qu'en RMN l'échantillon est préservé et peut ensuite servir à l'analyse par CPG-SM.

La RMN permet de détecter des substances responsables d'intoxication aiguë ou des substances prescrites en thérapeutique, quand les doses sont de l'ordre de plusieurs centaines de milligrammes. Mais, elle n'est apparemment pas utile quand les substances se retrouvent en très faibles quantités dans les liquides biologiques. La quantification est aisée mais la concentration doit être au moins de $10 \mu \mathrm{M}$ et de préférence entre 50 et $100 \mu \mathrm{M}$. 
Quand la RMN est disponible dans un laboratoire de clinique, il est possible de réaliser un screening rapide. Les résultats peuvent être obtenus en moins de 30 minutes.

La RMN a l'avantage de détecter simultanément des métabolites polaires et le composé parent, ce qui est difficile en CPG-SM. Un autre avantage est la détection de composés de faible poids moléculaire comme les acides formique ou glycollique, la détection de substances volatiles, de polymères solubles de haut poids moléculaire (65). Beaucoup plus facilement qu'en chromatographie, la RMN permet de distinguer des isomères ou des métabolites de structures très semblables (65).

La plupart des études ont été effectuées à $300 \mathrm{MHz}$. De tels instruments sont plus accessibles et moins chers que les appareils à haut champ $(400,600 \mathrm{MHz}$ ou plus) utilisés pour des études identiques $(67,71,82)$. Ces appareils augmentent la qualité des spectres en réduisant la superposition des signaux entre composés endogènes et xénobiotiques. Les spectres sont donc simplifiés et la sensibilité est augmentée pour détecter de plus faibles quantités de substances et de métabolites. Cependant toutes les capacités n'ont pas encore été étudiées et on pourrait imaginer un simple prétraitement chromatographique des échantillons avant l'analyse
RMN, ce qui permettrait de détecter plus facilement les composés. La connaissance des spectres RMN devrait permettre le développement de bases informatiques de spectres permettant une identification plus aisée des xénobiotiques.

Cette technique présente beaucoup d'avantages : rapidité, faible volume d'échantillon requis, quantification simple et détection simultanée de nombreux composés sans traitement de l'échantillon dans la plupart des cas. Le principal inconvénient de la RMN est son manque de sensibilité.. La limite de détection est beaucoup plus élevée qu'en CPG-SM et en conséquence des composés se trouvant à l'état de traces ne sont pas habituellement détectés. Cependant ce manque de sensibilité ne semble pas primordial en toxicologie d'urgence où les xénobiotiques et leurs métabolites sont présents en grande quantité et généralement associés à des symptômes cliniques.

L'EC et la RMN sont des techniques récentes, elles sont en pleine expansion. Que leurs réserve le futur ? Des couplages plus sophistiqués devraient leur donner un essor plus important. L'EC-SM est en cours de développement (17). Pour la RMN, on commence à décrire des couplages CLHP-RMN (83) et CLHP-RMN-SM (84). L'avenir devrait peut-être conduire à un couplage EC-SM-RMN.

\section{Références}

1. Moulsma M., Lardet G., Pascal P., Gagnieu M.C., Gaulier J.M., Vallon J.J. Dépistage immunologique des benzodiazépines dans le sang et les urines. Toxicorama $1996 ; 8: 23-8$.

2. Maurer H.H. Systematic toxicological analysis of drugs and their metabolites by gas chromatography- mass spectrometry. J. Chromatogr. 1992 ; 580 : 3-41.

3. Marquet P., Lachatre G. Liquid chromatography-mass spectrometry potential in forensic and clinical toxicology. J. Chromatogr. 1999 ; 733 : 93-118.

4. Boone C.M., Waterval J.C.M., Lingeman H., Ensing K., Underberg W.J.M., Capillary electrophoresis as a versatile tool for the bioanalysis of drugs - a review. J. Pharm. Biomed. Anal. $1999 ; 20: 831-63$.

5. Labat L., Deveaux M., Dubost J.P. Applications de l'électrophorèse capillaire en toxicologie clinique et médicolégale. Ann. Toxicol. Anal. 2000 ; XII (3) : 171-95.

6. Heitmeir S., Blaschke G., Direct assay of nonopiod analgesics and their metabolites in human urine by capillary electrophoresis and capillary electrophoresis-mass spectrometry. J. Chromatogr. B $1999 ; 721$ : 109-25.

7. Thormann W., Lienhard S., Wernly P., Strategies for the monitoring of drugs in body fluids by micellar electrokinetic capillary chromatography. J. Chromatogr. 1993 ; $636: 137-48$.
8. Wu W.S., Tsai J.L., Analysis of morphine and morphine3beta-D glucuronide in human urine by capillary zone electrophoresis with minimal sample pretreatment. Biomed. Chromatogr. 1999 ; 13 (3) : 216-9.

9. Hempel G., Strategies to improve the sensitivity in capillary electrophoresis for the analysis of drugs in biological fluids. Electrophoresis $2000 ; 21: 691-8$.

10. Couderc F., Caussé E., Bayle C., Drug analysis by capillary electrophoresis and laser-induced fluorescence. Electrophoresis $1998 ; 19: 2777-90$.

11. Wey A.B., Zhang C.X., Thormann W., Head-column field-amplified sample stacking in binary system capillary electrophoresis. Preparation of extracts for determination of opioids in microliter amounts of body fluids. J. Chromatogr. A 1999 ; 853 (1-2) : 95-106.

12. Lee K.J., Lee J.J., Moon D.C. Determination of tricyclique antidepressants in human plasma by micellar electrokinetic capillary chromatography. J. Chromatogr. $1993 ; 616: 135-43$.

13. Schafroth M., Thormann W., Allemann D., Micellar electrokinetic capillary chromatography of benzodiazepines in human urine. Electrophoresis $1994 ; 15$ (1) : 72-8. 
14. Caslavska J., Lienhard S., Thormann W. Comparative use of three electrokinetic capillary methods for the determination of drugs in body fluids. Prospects for rapid determination of intoxications. J. Chromatogr. $1993 ; 638$ : 335-42.

15. Taylor R.B., Low A.S., Reid R.G., Determination of opiates in urine by capillary electrophoresis. $\mathbf{J}$. Chromatogr. B Biomed. Appl. 1996 ; 675 (2) : 213-23.

16. Chee G.L., Wan T.S.M., Reproductible and high-speed separation of basic drugs by capillary zone electrophoresis. J. Chromatogr. $1993 ; 612: 172-7$.

17. Maurer H.H. Systematic toxicological analysis procedures for acidic drugs and/or metabolites relevant to clinical and forensic toxicology and/or doping control. J. Chromatogr. B Biomed. Sci. Appl; 1999 ; 733 (1-2) : 3-25.

18. Steimann L., Thormann W., Characterization of competitive binding, fluorescent drug immunoassays based on micellar electrokinetic capillary chromatography. Electrophoresis $1996 ; 17: 1348-56$.

19. Thormann W., Aebi Y., Lanz M., Caslavska J., Capillary electrophoresis in clinical toxicology. Forensic Sci. Int. 1998 ; 92 (2-3) : 157-83.

20.Pucci V., Raggi M., Kenndler E., Separation of eleven central nervous system drugs by capillary zone electrophoresis. J. Chromatogr. B Biomed. Sci. Appl. 1999 ; 728 (2) : 263-71.

21.Boone C.M., Franke J.P., de Zeeuw R.A., Ensing K., Evaluation of capillary electrophoresis techniques towards systematic toxicological analysis. J. Chromatogr. A $1999 ; 838(1-2)$ : 259-72.

22. Hudson J., Golin M., Malcolm M., Whiting C., Capillary zone electrophoresis in a comprehensive screen for drugs of forensic interest in whole blood : an update. Can. Soc. Forens. Sci. J. $1998: 31$ (1) : 1-29.

23.Boone C.M., Franke J.P., de Zeeuw R.A., Ensing K., Intra- and interinstrument reproducibility of migration parameters in capillary electrophoresis for substance identification in systematic toxicological analysis. Electrophoresis $2000 ; 21: 1545-51$.

24. Dehon B., Lhermitte M., Cyanures et anions. In : Toxicologie et pharmacologie médicolégales. Kintz P., Elsevier, Paris, 1998 ; 147-87.

25. Shihabi Z.K., Friedberg M.A., Analysis of small molecules for clinical diagnosis by capillary electrophoresis. Electrophoresis $1997 ; 18$ : 1724-32.

26. Fritz J.S., Recent developments in the separation of inorganic and small organic ions by capillary electrophoresis. J. Chromatogr. A $2000 ; 884$ (1-2) : 261-75.

27. Krivacsky Z., Gelencser A., Hlavay J., Kiss G., Sarvari $Z$., Electrokinetic injection in capillary electrophoresis and its application to the analysis of inorganic compounds. J. Chromatogr. A 1999 ; 834 (1-2) : 21-44.

28. Zunic G., Spasic S., Jelic-Ivanovik Z., Simple and rapid method for the measurement of nitrite and nitrate in human plasma and cerebrospinal fluid by capillary electrophoresis. J. Chromatogr. B Biomed. Sci. Appl. 1999 ; $727(1-2):$ 73-9.

29. Bories P.N., Scherman E., Dziedzic L., Analysis of nitrite and nitrate in biological fluids by capillary electrophoresis. Clin. Biochem. 1999 ; 32 (1) : 9-14.
30. Macka M., Haddad P.R., Determination of metal ions by capillary electrophoresis. Electrophoresis $1997 ; 18$ : 2482-501.

31. Thormann W., Wey A.B., Lurie I.S., Gerber H., Byland C., Malik N., Hochmeister M., Gehrig C., Capillary electrophoresis in clinical and forensic analysis : recent advances and breakthrough to routine applications. Electrophoresis 1999 ; 20 (15-16) : 3203-36.

32. Frost M., Köhler H., Blaschke G., Enantioselective determination of methadone and its main metabolite 2ethydene-1,5-dimethyl-3,3-diphenylpyrrolidine (EDDP) in serum, urine and hair by capillary electrophoresis. Electrophoresis $1997 ; 18$ : 1026-34.

33.Jumpannen J., Siren H., Riekkola M.L., Screening for diuretics in urine and blood serum by capillary zone electrophoresis. J. Chromatogr. A 1993 ; 652 : 441-50.

34. Lukkari P., Nyman T., Riekkola M.L., Determination of nine beta-blockers in serum by micellar electrokinetic capillary chromatography. J. Chromatogr. A 1994 ; 674 : 241-6.

35. Steimann L., Thormann W., Toxicological drug screening and confirmation by electrokinetic capillary techniques : Concept of an automated system. J. Cap. Electrophoresis $1995 ; 2: 81-8$.

36. Caslavska J., Allemann D., Thormann W., Analysis of urinary drugs by a multianalyte capillary electrophoretic immunoassay. J. Chromatogr. A 1999 ; 838 (1-2) : 197 211.

37. Ramseier A., Caslavska J., Thormann W., Screening for urinary amphetamine and analogs by capillary electrophoretic immunoassays and confirmation by capillary electrophoresis with on-column multiwavelength absorbance detection. Electrophoresis 1998 ; 19 : 2956-66.

38. Heitmeier S., Blaschke G. Direct determination of paracetamol and its metabolites in urine and serum by capillary electrophoresis with ultraviolet and mass spectrometric detection. J. Chromatogr. B Biomed. Sci. Appl. 1999 ; 721 (1) : 93-108.

39. Ramseier A., Siethoff C., Caslavska J., Thormann W. Confirmation testing of amphetamines and designer drugs in human urine by capillary electrophoresis-ion trap mass spectrometry. Electrophoresis $2000 ; 21: 380-7$.

40. Tsai J.L., Wu W.S., Lee H.H., Qualitative determination of urinary morphine by capillary zone electrophoresis and ion trap mass spectrometry. Electrophoresis 2000 ; $21: 1580-6$

41. McClean S., O'Kane E.J., Hillis J., Smyth W.F., Determination of 1,4-benzodiazepines and their metabolites by capillary electrophoresis and high-performance liquid chromatography using ultraviolet and electrospray ionisation mass spectrometry. J. Chromatogr. A 1999 ; $838(1-2)$ : 273-91.

42. McClean S., O'Kane E.J., Smyth W.F. The identification and determination of selected 1,4-benzodiazepines by an optimised capillary electrophoresis-electrospray mass spectrometric method. Electrophoresis $2000 ; 21$ : 1381-9.

43. Cherkaoui S., Veuthey J.L., Development and robustness testing of a nonaqueous capillary electrophoresis method for the analysis of nonsteroidal antiinflammatory drugs. J. Chromatogr. A $2000 ; 874$ (1) : 121-9. 
44. Harris R.K. Nuclear Magnetic Resonance Spectroscopy. 1986 ; Longman Scientific \& Technical.

45. Nicholson J.K. Foxall J.D., Spraul M., Farrant R.D., Lindon J.C. $750 \mathrm{MHz} 1 \mathrm{H}$ and 1H-13C NMR spectroscopy of human blood plasma. Anal. Chem. 1995 ; 67 : 793-811.

46. Malet-Martino M.C., Servin P., Bernadou J., Armand J.P., Martino R. Human urinary excretion of doxifluridine and metabolites during a 5-day chemotherapeutic schedule using fluorine-19 nuclear magnetic resonance spectrometry. Investig. New Drugs. 1987 ; 5 : 273-9.

47. Preece N.E., Challands J., Williams S.C. 19F NMR studies of enflurane elimination and metabolism. NMR Biomed. 1992 ; 5 : 101-6.

48. Nicholson J.K., Wilson I.D. High resolution proton magnetic resonance spectroscopy of biological fluids. Prog. NMR Spectrosc. 1989 ; 21 : 449-501.

49. Bell J.D., Preece N.E., Parkes H.G. NMR studies of body fluids extracts. In NMR in Physiology and Biomedicine, ch 14, Gilies R.J., Ed. Academic Press, New York, NY, 1994, pp 221-36.

50.Zuppi C., Messane F., Forni F., Rossi L., Pennacchietti L., Ferrari F., Giardinia G. 1H NMR Spectra of normal urines : reference ranges of the major metabolites. Clin. Chim. Acta. 1997 ; 265 : 85-97.

51. Yamaguchi S., Koda N., Eto Y., Aoki K. Quick screening and diagnosis of organic acidemia by NMR urinalysis. J. Pediatr. $1985 ; 106: 620-2$.

52. Lehnert W., Hunkler D. Possibilities of selective screening for inborn errors of metabolism using high-resolution 1H-FT-NMR spectrometry. Eur. J. Pediatr. 1986 ; $145: 260-6$.

53. Bock J.L. NMR in clinical chemistry-Where do we stand? Clin. Chem. $1994 ; 40 ; 56-61$.

54. De Certaines J.D. High resolution magnetic resonance spectroscopy in clinical biology. Leukemia. 1990; 4 : $667-70$

55. Eugene M., Spectrométrie RMN des liquides biologiques. Rean. Urg. $1993 ; 2$ : 605-8.

56. Wevers R.A., Engelke U., Heerschap A. High resolution 1H-NMR spectroscopy of blood plasma for metabolic studies. Clin. Chem. $1994 ; 40: 1245-50$.

57. Vion-Dury J., Favre R., Sciaky M., Kriat M., ConfortGouny S., Harl J.R., Grazziani N., Viout P., Cozzone P.J. Graphic-aided study of metabolic modifications of plasma in cancer using proton magnetic resonance spectroscopy. NMR Biomed. $1993 ; 6$ : 58-65.

58. Eugene M.. Le Moyec L., De Certaines J.D., Desruennes M., Le Rumeur E., Fraysse J.B., Cabrol C. Lipoproteins in heart transplantation : proton magnetic resonance spectroscopy of plasma. Magn. Reson. Med. 1991; 18 : 93-101.

59. Pont H., Vion-Dury J., Kriat M., Mouly-Bandini A., Sciaky M., Viout M., Confort-Gouny S., Messana T., Goudart M., Monties J.R., Cozzone P.J. NMR spectroscopy of plasma during acute rejection of transplanted hearts. Lancet. 1991 ; 337 : 792-3.

60. Ghaury F.Y.K., Blackledge C.A., Wilson I.D., Nicholson J.K. Studies on metabolism of fluorinated xenobiotics in rats using 19F-NMR and 1H-NMR spectroscopy. J. Pharm. Biomed. Anal. $1990 ; 8$ : 939-44.
61.Spurway T.D., Gartland K.P.R., Warrander A., Pickford R., Nicholson J.K., Wilson I.D. Proton nuclear magnetic resonance of urine and bile from paracetamol dosed rats. Pharm. Biomed. Anal. $1990 ; 8$ : 969-73.

62. Gartland K.P.R., Bedell C.R., Lindon J.C., Nicholson J.K Application of pattern recognition methods to the analysis and classification of toxicological data derived from proton nuclear magnetic resonance spectroscopy of urine. Mol. Pharmacol. 1991 ; 39 : 629-42.

63. Bales J.R., Bell J.D., Nicholson J.K., Sadler P.J., Timbrell J.A., Hughes R.D., Bennett P.N., Williams R. Metabolic profiling of body fluids by proton NMR: selfpoisoning episodes with paracetamol (acetaminophen). Magn. Reson. Med. 1988 ; 6 : 300-6.

64. Wilson I.D., Nicholson J.K. Solid phase extraction chromatography and NMR spectroscopy (SPEC-NMR) for the rapid identification of drug metabolites in urines. J. Pharm. Biomed. Anal. $1988 ; 6$ : 151-65.

65. Komoroski E.M., Komoroski R.A., Valentine J. L., Pearce J., Kearns G.L. The use of nuclear magnetic resonance spectroscopy in the detection of drug intoxication. J. Anal. Toxicol. $2000 ; 24: 180-7$.

66. Maschke S., Azaroual N., Imbenotte M., Vermeersch G., Leclerc F., Lhermitte M. Salicylate poisoning : twodimensional J-resolved NMR urinalysis. NMR Biomed. $1995 ; 8: 19-24$.

67. Maschke S., Azaroual N., Wieruszeeski J.M., Lippens G., Imbenotte M., Mathieu D., Vermeersch G., Lhermitte $\mathrm{M}$. Diagnosis of a case of acute chloroquine poisoning using 1H-NMR spectroscopy : characterization of drug metabolites in urine. NMR Biomed. $1997 ; 10: 277-84$.

68. Bales J.R., Sadler P.J., Nicholson J.R., Timbrell J.A. Urinary excretion of acetaminophen and its metabolites as studied by proton NMR spectroscopy. Clin. Chem. $1984 ; 30: 1631-6$.

69. Nicholls A.W., Caddick S., Wilson I.D., Farrant R.D., Lindon J.C., Nicholson J.K. High resolution NMR spectroscopic studies on the metabolism and futile deacetylation of 4-hydroxyacetanilide (paracetamol) in the rat. Biochem. Pharmacol. 1995 ; 49 : 1155-64.

70. Vermeersch G., Marko J., Cartigny B., Leclerc F., Roussel P., Lhermitte M. Salicylate poisoning detected by $1 \mathrm{H}-$ NMR spectroscopy. Clin. Chem. 1988 ; 34 : 1003-4.

71. Maschke S., Azaroual N., Wieruszeeski J.M., Lippens G., Imbenotte M., Mathieu D., Vermeersch G., Lhermitte M. Detection by $1 \mathrm{H}-\mathrm{NMR}$ spectroscopy of chloroquine in urine from acutely poisoned patient. Clin. Chem. 1997 ; 43 : 698-9.

72. Azaroual N., Imbenotte M., Cartigny B., Leclerc F., Vallee L., Lhermitte M., Vermeersch G. Valproic acid intoxication identified by $1 \mathrm{H}$ and $1 \mathrm{H}-13 \mathrm{C}$ correlated NMR spectroscopy of urine samples. MAGMA. 2000 ; $10: 177-82$

73. Azaroual N., Imbenotte M., Cartigny B., Vallee L., Lhermitte M., Vermeersch G. Analysis of two cases of valproic acid intoxication by $1 \mathrm{H}$ NMR spectroscopy and gas chromatography-mass spectrometry. Acta Clin. Belg. $1999 ; 1: 25-8$.

74. Everett J.R. Spin-echo 1H-NMR spectroscopy : a new method for studying penicillin metabolism. J. Chem. Soc. 1984 ; 894-5. 
75. Wahl A., Azaroual N., Imbenotte M., Mathieu D., Forzy G., Cartigny B., Vermeersh G., Lhermitte M. Poisoning with methanol and ethylene glycol : 1H-RMN spectroscopy as an effective clinical tool for diagnosis and quantification. Toxicology. $1998 ; 128: 73-81$.

76. Pappas A.A., Thompson J.R., Fuller G.L., Porter .H., Gadsen R.H. High-resolution proton nuclear magnetic resonance spectroscopy in the detection of low molecular weight volatiles. J. Anal. Toxicol. 1993 ; 17 : 273-7.

77. Cartigny B., Azaroual N., Imbenotte M., Sadeg N., Testart F., Richecoeur J., Vermeersch G., Lhermitte M. Intoxication aiguë au tétrahydrofuranne : analyse de liquides biologiques par spectroscopie RMN $1 \mathrm{H}$. Toxicorama. $999 ; 11: 250-4$.

78. Imbenotte M., Azaroual N., Mathieu D., Cartigny B., Vermeersch G., Lhermitte M. Determination by $1 \mathrm{H}-$ NMR spectroscopy of paraquat in urine from acutely poisoning patients. Comparison with second-derivative spectroscopy method. J. Anal. Toxicol. $1999 ; 23$ : 58690.

79. Wahl A., Azaroual N., Imbenotte M., Mathieu D., Vermeersch G., Mereau C., Lhermitte M. Identification et quantification du glyphosate dans les fluides biologiques par RMN 1H et 31P. Toxicorama. 1997 ; 9 numéro spécial : $340-5$
80. Brown J.C.C., Mills G.A., Sadler P.J., Walker V. 1HNMR studies of urine from premature and sick babies. Magn. Reson. Med. 1989 ; 11 : 193-201.

81. Meshitsuka S., Inoue M., Seki A., Koeda T., Takeshita K. Screening of urine by one-dimensional and pulsed-field gradient two-dimensional $1 \mathrm{H}$ NMR spectroscopy : intoxication by polypropylene glycol in an infant patient. Clin. Chim. Acta. 1999 ; $279: 47-54$.

82. Foxall P.J.D., Parkinson J.A., Sadler I.H., Lindon J.C., Nicholson J.K. Analysis of biological fluids using 600 $\mathrm{MHz}$ proton NMR spectroscopy : application of homonuclear two-dimensional $j$-resolved spectroscopy to urine and blood plasma for spectral simplification and assignment. J. Pharmaceut. Biomed. Anal. 1993 ; 11 : 21-31.

83. Lindon J.C., Nicholson J.K., Sidelmann U.G., Wilson I.D. Directly coupled HPLC-NMR and its application to drug metabolism. Drug Metab. Rev. 1997 ; 29 : 705-46.

84. Scarfe G.B., Lindon J.C., Nicholson J.K., Martin P., Wright B., Taylor S.D. Investigation of the metabolism of $14 \mathrm{C} / 13 \mathrm{C}$-practolol in rat using directly coupled radioHPLC-NMR-MS. Xenobiotica. 2000 ; 30 : 717-29. 